\title{
Propiedades Psicométricas de la Escala de Comportamiento Preescolar y Jardín Infantil en una Muestra de Niños Argentinos de 3 a 7 Años
}

\section{Psychometric Properties of the Preschool and Kindergarten Behavior Scales in a Sample of Argentinean Children from 3 to 7 Years Old}

\author{
Cecilia Reyna y Silvina Brussino \\ Universidad Nacional de Córdoba
}

\begin{abstract}
El objetivo de este estudio fue analizar las propiedades psicométricas de la Escala de Comportamiento Preescolar y Jardín Infantil en una muestra de niños argentinos de 3 a 7 años, y obtener una versión reducida de la escala con adecuadas propiedades psicométricas para ser utilizada como instrumento de screening. Para el análisis factorial exploratorio se utilizó una muestra de 208 niños de ambos sexos y el análisis confirmatorio se realizó en base a una muestra de 184 niños, también de ambos sexos. Se obtuvieron estructuras factoriales semejantes a las obtenidas en estudios sobre la versión original, con menor cantidad de reactivos. En general, las escalas de Habilidades Sociales y Problemas de Conducta mostraron buenos índices de confiabilidad.
\end{abstract}

Palabras clave: habilidades sociales, problemas de conducta, niños, instrumento de evaluación.

\begin{abstract}
The goal of this study was to analyze the psychometric properties of the Preschool and Kindergarten Behavior Scales in a sample of Argentinean children from 3 to 7 years old, and to obtain a reduced version of the scale with adequate psychometric properties for use as a screening tool. An exploratory factor analysis was performed in a sample of 208 children of both sexes whereas the confirmatory analysis was conducted on a sample of 184 children, also of both sexes. Factor structures that emerged from this study were similar to those obtained with the original version in other studies, with fewer items. In general, the scales of Social Skills and Behavior Problems showed good reliability.
\end{abstract}

Keywords: social skills, behavior problems, children, evaluation tool.

Introducción

En las últimas décadas se incrementó de manera notoria el interés por el desarrollo de las habilidades sociales en niños, tanto en el campo de la psicopatología como en el estudio del desarrollo normal. Problemas de adaptación en la niñez se han asociado con dificultades posteriores, como abandono escolar, criminalidad (Parker \& Asher, 1987) y abuso de drogas (Patterson, DeGarmo \& Knutson, 2000).
Si bien ninguna definición de competencia social es aceptada de manera unívoca, se reconoce que los individuos competentes a nivel social poseen repertorios de conductas adecuadas socialmente y habilidades de resolución de problemas que les permiten elegir y llevar a cabo ciertas conductas en situaciones de interacción social (Cummings, Kaminski \& Merrell, 2008), conductas organizadas que se evidencian en distintos momentos de la vida (Rose-Krasnor, 1997) y se

Cecilia Reyna, Laboratorio de Psicología Cognitiva, Facultad de Psicología, Universidad Nacional de Córdoba, Argentina. Becaria de Postgrado Tipo I del Consejo Nacional de Investigaciones Científicas y Tecnológicas, Argentina.

Silvina Brussino, Laboratorio de Psicología Cognitiva, Facultad de Psicología, Universidad Nacional de Córdoba, Argentina. Investigador de Carrera del Consejo Nacional de Investigaciones Científicas y Tecnológicas, Argentina. La correspondencia relativa a este artículo debe ser dirigida a Cecilia Reyna, Laboratorio de Psicología Cognitiva, Facultad de Psicología, Universidad Nacional de Córdoba, Enfermera Gordillo esquina Enrique Barros, Ciudad Universitaria, 5000 Córdoba, Argentina. E-mail: cereyna@psyche.unc.edu.ar

Las autoras agradecen la colaboración de los estudiantes Constanza Bracco, Julia Ipiña, Martín Izaguirre, Gabriela Lello, Lucas Narambuena, Damián Revillo y Anahí Sánchez, por su contribución en la recolección de datos en la ciudad de Córdoba, y a las instituciones educativas, padres y docentes que participaron en este estudio. 
relacionan con estructuras motivacionales y afectivas (Trianes, Muñoz \& Jiménez, 1997). Gresham, Sugai y Horner (2001) señalan que la competencia social es un concepto evaluativo sobre el desempeño social de un individuo, basado en el juicio de padres, maestros o pares. Dentro de ese marco, las habilidades sociales se consideran comportamientos específicos que permiten al individuo desempeñarse en un contexto interpersonal y manifestar de manera adecuada sentimientos, actitudes, deseos, opiniones o derechos, respetando dichas conductas en los demás, lo que favorece la resolución de problemas inmediatos y minimiza problemas futuros (Caballo, 2005). Ambos conceptos se enmarcan dentro del comportamiento adaptativo del individuo.

Mientras que las habilidades sociales se asocian con un comportamiento adaptativo, los problemas de conducta se relacionan con comportamientos no adaptativos. Dentro de estos últimos, se distinguen dos grandes categorías: problemas externalizantes, que se caracterizan por agresión, pelea y acting-out, y problemas internalizantes, como ansiedad, aislamiento social y depresión. Por otra parte, en una categorización general de habilidades sociales se considera, por un lado, la relación con pares y, por otro, la relación con adultos. Caldarella y Merrell (1997), en una revisión cualitativa de investigaciones que reportan escalas de habilidades sociales, encontraron de manera consistente la presencia de cinco dimensiones: relación con pares, manejo propio, académica, conformidad y asertividad, observando solapamiento entre dimensiones, lo cual implica que no son completamente independientes unas de otras.

En un estudio reciente sobre salud mental en niños de 6 a 11 años, llevado a cabo en ocho centros urbanos de Argentina en una muestra de 5.697 niños, se observó que el $14,6 \%$ presenta problemas internalizantes y el $14,9 \%$, problemas externalizantes (más de una desviación estándar superior a la media), lo cual es considerado en el estudio en una categoría de vulnerabilidad alta o muy alta (Argentina, Ministerio de Salud de la Nación y Unidades Académicas de Psicología de Universidades Nacionales, 2007). Estos indicadores señalan la necesi- dad de una pronta respuesta a los problemas de comportamiento social infantil, tanto desde la atención como de la prevención y promoción temprana de conductas adaptativas, para lo cual se requiere contar con adecuados instrumentos de evaluación y programas de intervención.

Entre las técnicas de evaluación del comportamiento social, los cuestionarios son los más utilizados, los cuales comprenden medidas de auto-reporte y reporte de padres o maestros, siendo los auto-reportes escasamente utilizados con niños de edad preescolar. Las escalas de comportamiento de Achenbach (2001, citado en Merrell \& Harlacher, 2008 [Child Behavior Checklist, CBCL, Listado de Comportamientos Infantiles]) son las más utilizadas, aunque han sido diseñadas como instrumentos de evaluación psicopatológica, por lo cual pueden no ser útiles para evaluar las conductas sociales habituales de los niños y para situaciones de screening breves debido a que son muy extensas. Matson y Wilkins (2009) revisaron 40 tests de habilidades sociales para niños y señalan que los instrumentos de mayor uso son el Matson Evaluation of Social Skills with Youngsters ([MESSY, Evaluación de Habilidades Sociales para Jóvenes], Matson, Rotatori \& Helsel, 1983) y el Social Skills Rating System ([SSRS, Sistema de Evaluación de Habilidades Sociales], Gresham \& Elliott, 1990, citado en Matson \& Wilkins, 2009). El MESSY se utiliza principalmente con niños de edad escolar y adolescentes y también en poblaciones de individuos bipolares, autistas y con deficiencia visual. Por su parte, el SSRS se utiliza más en el contexto escolar. Desde un punto de vista evolutivo, ambas escalas son más generales que otros instrumentos como, por ejemplo, el Preschool and Kindergarten Behavior Scales ([PKBS, Escala de Comportamiento Preescolar y Jardín Infantil], Merrell, 1996).

El PKBS es una de las escalas que combina buenas propiedades psicométricas con contenido específico para niños pequeños, sin ser una extensión de escalas originalmente diseñadas para niños de edad escolar. La versión original cuenta con estudios normativos basados en una muestra nacional de niños estadounidenses de 3 a 6 años 
evaluados por padres y docentes (Merrell, 1996). Presenta una alta consistencia interna y una estabilidad temporal adecuada, sobre todo considerando las fluctuaciones de períodos tempranos del desarrollo infantil (Carney \& Merrell, 2005), y posee buenas características de validez de constructo (Merrell, 1996). Además, ha mostrado validez convergente y discriminante en relación a escalas de evaluación de hiperactividad (Canivez \& Bordenkircher, 2002; Canivez \& Rains, 2002), otras escalas de comportamiento social (Jentzsch \& Merrell, 1996) y medidas de observación conductual (Winsler \& Wallace, 2002). Asimismo, ha mostrado ser una herramienta útil para diferenciar a niños con potencial retraso de desarrollo y grupos de comparación (Holland \& Merrell, 1998; Jentzsch \& Merrell, 1996; Merrell \& Wolfe, 1998) y a niños con problemas internalizantes en relación a niños con desarrollo típico (Merrell \& Wolfe, 1998). Merrell (1996, 2000) señala la utilidad de la escala como instrumento de screening, permitiendo identificar a niños con un riesgo potencial de desarrollar problemas emocionales, sociales o conductuales, con 1,5 desviaciones estándar por debajo de la media normativa en habilidades sociales y/o por arriba de la media normativa en problemas de conducta, revelando la necesidad de una evaluación más amplia. La escala también puede utilizarse en la fase de evaluación, pero solo en conjunto con otros instrumentos y fuentes de evaluación. Además, se reconoce su utilidad en la generación de metas de intervención.

En consideración a la creciente población hispana en Estados Unidos, Carney y Merrell (2002) elaboraron una versión en español y, con el fin de estudiar la relación entre las versiones en inglés y español, las aplicaron a cuidadores bilingües de niños entre 3 y 6 años. Las versiones mostraron estar correlacionadas de manera significativa ( $r$ de Pearson 0,93 para la escala de Habilidades Sociales y 0,94 para Problemas de Conducta, $p<0,001)$ y la consistencia interna, según el coeficiente alfa de Cronbach, fue semejante (Habilidades Sociales =0,93, Problemas de Conducta $=0,96)$. Posteriormente, Merrell (2003) presenta la segunda edición de la escala en español, sin contar con más estudios psicométricos que los señalados.

Si bien se recomienda utilizar múltiples fuentes y técnicas para caracterizar la conducta infantil, cuestiones de orden práctico (personal capacitado y aspectos económicos, entre otros) hacen que en pocas ocasiones se recurra a más de un informante. Como señalan Matson y Wilkins (2009), en 33 de 41 estudios que utilizan tres de las escalas más estudiadas (MESSY, SSRS y List of Social Situation Problems [Lista de Problemas de Situaciones Sociales]) se recurre a un informante, y en ocho estudios, a un padre/madre y un docente. Por otra parte, Caldarella y Merrell (1997) encontraron que los maestros fueron los informantes en el 71,4\% de los estudios revisados y los padres en el 19,1\%; se recurrió al auto-reporte en el 19,1\% de las investigaciones y a la evaluación sociométrica de pares en el $4,8 \%$, mientras que el $14,3 \%$ de las investigaciones utilizaron dos técnicas de recolección de datos. Un estudio que utiliza las escalas de Achenbach para padres y maestros pone de manifiesto que las evaluaciones de los maestros resultan ser las mejores referencias para los servicios de salud mental (Stanger \& Lewis, 1993). En el mismo sentido, en otro estudio (Bank, Duncan, Patterson \& Reid, 1993) el informe de los maestros resultó ser el mejor predictor de problemas de delincuencia y arrestos. Tal como mencionan Canivez y Rains (2002), los docentes son fuentes importantes de información sobre la conducta del niño debido a que son observadores naturales e informantes en situaciones escolares, contando con una relevante experiencia comparativa en observar a los niños en distintos momentos y contextos sociales.

En Argentina, los instrumentos de evaluación del comportamiento infantil se focalizaban primordialmente en los problemas de conducta y en niños de edad escolar, como la adaptación y validación del CBCL realizada por Samaniego (1998) y la Guía de Observación Comportamental para Niños (Ison \& Facchinelli, 1993; Ison \& Soria, 1997). Con respecto a las habilidades sociales, laEscaladeComportamientoAsertivo ha sido adaptada y normalizada por Brussino (2002) en niños de 6 a 12 años, mientras que el MESSY cuenta con estudios realizados 
por Schulz (2008) en niños de 8 a 12 años, con buenos resultados de confiabilidad y validez. Recientemente, Lacunza, Castro Solano y Contini (en prensa) construyeron una Escala de Habilidades Sociales para niños preescolares a partir de ítems de diversos cuestionarios, entre los que se encuentra el PKBS. La escala se aplica a padres de niños de 3 a 5 años y comprende aspectos referidos a habilidades sociales, con un protocolo distinto para cada edad, obteniéndose solo una puntuación total, ya que estudios sobre su estructura factorial mostraron una difícil interpretación de los eventuales factores. También se reportaron adecuados índices de consistencia interna.

En función de la revisión de antecedentes y teniendo en cuenta la necesidad de contar con instrumentos adaptados a la población argentina para evaluar el comportamiento social infantil, sobre todo en edades tempranas, el objetivo de este estudio fue analizar las características psicométricas de la Escala de Comportamiento Preescolar y Jardín Infantil (Merrell, 2003), focalizándose en la revisión de la estructura interna de la escala y en su confiabilidad, a través de la estimación de la consistencia interna. Asimismo, tuvo como propósito contar con una versión reducida de la escala con el fin de hacer más sencilla su interpretación cuando se utiliza en conjunto con otras técnicas.

\section{Método}

\section{Participantes}

La selección de los participantes se realizó de manera no aleatoria debido a las posibilidades de acceso a la población de interés. Se contactó con distintas instituciones $\mathrm{y}$ se incluyeron aquellas que accedieron a participar. Para el análisis factorial exploratorio se recurrió a una muestra de 208 niños de 3 a 7 años (45,2\% varones y $54,8 \%$ mujeres) de la ciudad de Mendoza que asistían a instituciones educativas públicas y privadas. El análisis factorial confirmatorio se llevó a cabo en base a una muestra de 184 niños de 5 y 7 años (40,8\% varones y $59,2 \%$ mujeres) que asistían a instituciones públicas de la ciudad de Córdoba. Cabe señalar que las ciudades de Mendoza y Córdoba poseen características semejantes en cuanto a aspectos socioeconómicos y demográficos. Los niños de ambas muestras pertenecían a distintos niveles socioeconómicos (NSE): 9,4\% marginal, $15,5 \%$ bajo inferior, $32,3 \%$ bajo superior, $10,3 \%$ medio inferior, $12,1 \%$ medio típico, 4,4\% medio alto y $16 \%$ alto. Ninguno de los niños se encontraba bajo tratamiento médico/psicológico ni tomaba medicación.

\section{Instrumentos}

Cuestionario Sociodemográfico. A través de un cuestionario estructurado aplicado a los padres, se obtuvo información sobre el NSE: ocupación y nivel educativo del principal sostén del hogar, cantidad de aportantes y miembros del hogar, cobertura de salud e indicadores de indigencia.

\section{Escala de Comportamiento Preescolar y} Jardín Infantil. Se utilizó la segunda edición de la versión en español de esta escala (Merrell, 2003). Evalúa habilidades sociales y problemas de conducta en niños de 3 a 6 años, a través del informe de padres o maestros. La escala de Habilidades Sociales (34 ítems) comprende las subescalas de Cooperación Social (conductas cooperativas y de auto dominio), Interacción Social (inicio de conductas sociales) e Independencia Social (conductas para lograr independencia en el grupo de pares); y la escala de Problemas de Conducta (42 ítems), las subescalas de Problemas Externalizantes e Internalizantes. En la subescala de Problemas Externalizantes, a su vez, se pueden distinguir tres escalas: egocéntrico/explosivo, problemas de atención/hiperactividad y antisocial/agresivo; mientras que en Problemas Internalizantes se distinguen dos escalas: aislamiento social y ansiedad/ problemas somáticos. La conducta del niño se clasifica en una escala tipo Likert de 4 puntos (que oscila entre $0=$ nunca y $3=$ frecuentemente). Una mayor puntuación en la escala o subescalas de Habilidades Sociales indica un mejor desempeño, mientras que en la escala o subescalas de Problemas de Conducta señala comportamientos sociales no adaptativos. 
En 1996, Merrell reportó resultados de las propiedades psicométricas de la primera edición de la escala. Las escalas de Habilidades Sociales y Problemas de Conducta fueron analizadas por separado a través de análisis de componentes principales con rotación Varimax. Para la escala de Habilidades Sociales, Merrell obtuvo tres factores, Cooperación Social, Interacción Social e Independencia Social, que explicaron el $54 \%$ de la varianza total de las habilidades sociales. Para estudiar la estabilidad de la estructura de la escala realizó un análisis factorial confirmatorio, con estimación de mínimos cuadrados no ponderada, a partir de la cual obtuvo una estructura muy estable. Con respecto a la escala de Problemas de Conducta, obtuvo dos factores, Problemas Externalizantes y Problemas Internalizantes, que explicaron el $46 \%$ de la varianza total de los problemas de conducta. El análisis factorial confirmatorio indicó una alta estabilidad factorial. Las diferentes escalas mostraron buenos índices de consistencia interna (alfa de Cronbach osciló entre 0,88 y 0,97). Como se señaló en la introducción, la escala posee buenas estimaciones de estabilidad temporal y gran soporte de validez, contando ya con una segunda versión en español (Merrell, 2003), aunque sin más estudios psicométricos que los mencionados.

\section{Procedimiento y Análisis de Datos}

En primer lugar, cuatro docentes (dos de segundo grado [7 años], una de sala de 5 años y una de 3 años) evaluaron la comprensión de los ítems de la Escala de Comportamiento Preescolar y Jardín Infantil. La incorporación de docentes de los niveles mencionados se realizó teniendo en cuenta los estudios originales de la escala y las edades a las cuales se espera aplicar el instrumento en estudios posteriores (3 a 7 años). Luego de algunas modificaciones lingüísticas, se conformó la versión a analizar.

Una vez recibida la autorización escrita de los padres, los docentes de los niños autorizados completaron la escala en base a la observación del comportamiento del niño en el contexto de la escuela o jardín. Por otra parte, los padres completaron el cuestiona- rio sociodemográfico en pequeños grupos (máximo 4 personas) o individualmente, en horario y espacio escolar. En caso de no poder asistir a la institución, se acordó una visita al hogar. Tanto los padres como los docentes participaron de manera voluntaria. Se les informó previamente los objetivos del estudio así como el carácter anónimo y confidencial de la información brindada. Posterior a la recolección de datos, se llevaron a cabo los análisis estadísticos. Se realizaron estudios descriptivos de los ítems y luego se analizó de manera exploratoria la estructura factorial de las escalas en base a la muestra de niños de la ciudad de Mendoza; además, se estimó la consistencia interna. Para verificar el ajuste estadístico de los modelos se realizaron análisis confirmatorios, con la muestra de niños de la ciudad de Córdoba. Se utilizaron los paquetes estadísticos SPSS 17, FACTOR 7.02 y LISREL 8.51.

\section{Resultados}

En primer lugar, se inspeccionó la existencia de casos atípicos univariados a través del cálculo de puntuaciones estándar. Se consideraron atípicos los casos con puntuaciones $Z$ absolutas mayores a \pm 3 . También se examinó la presencia de casos atípicos multivariados, a través de la prueba de distancia de Mahalanobis $(p<0,001)$ (Hair, Anderson, Tatham \& Black, 1999). Producto de esos análisis, 16 casos resultaron atípicos. Tras verificar que la recolección de esos datos había sido anormal (principalmente, notable desinterés del docente), se decidió su eliminación. No se observaron variables con valores omitidos.

Se revisaron los ítems incluidos en cada una de las escalas. Se eliminó un ítem debido a la dificultad de los maestros para responderlo ("Desafía a sus padres, profesores o cuidadores" porque el término "desafía" resultó poco claro). Además, cinco ítems fueron descartados porque se asociaban más a otros constructos teóricos, tales como atención, control inhibitorio, estado emocional y recreación ("Se mantiene sentado/a escuchando cuando alguien está leyendo cuentos", "Tiene dificultad para concentrarse o permanecer en una tarea", "Demuestra autocontrol", "Cambia de estado de ánimo", 
"Usa el tiempo libre de manera adecuada"). Cabe señalar que los estudios psicométricos sobre este cuestionario se realizan en el marco de una investigación sobre desarrollo social infantil que comprende aspectos emocionales y cognitivos, por lo cual se procuró evitar el solapamiento de constructos.

Posterior a ello se analizó la asimetría y curtosis de la distribución de los ítems (Tablas 1 y 2). Los resultados obtenidos respondieron a lo esperado: los ítems de la escala de Habilidades Sociales presentaron

Tabla 1

Media, Desviación Estándar, Asimetría y Curtosis de la Distribución de los Ítems de la Escala de Habilidades Sociales

\begin{tabular}{|c|c|c|c|c|}
\hline Ítem & $M$ & $D E$ & Asimetría & Curtosis \\
\hline 1. Juega de forma independiente. & 2,36 & 0,81 & $-0,88$ & $-0,55$ \\
\hline 2. Es cooperativo/a. & 2,51 & 0,68 & $-1,05$ & $-0,13$ \\
\hline 3. Sonríe y ríe con los otros niños. & 2,84 & 0,41 & $-2,60$ & 6,39 \\
\hline 4. Juega con diferentes niños. & 2,72 & 0,50 & $-1,49$ & 1,28 \\
\hline $\begin{array}{l}\text { 5. Intenta comprender el comportamiento de otro niño (“¿por qué } \\
\text { lloras?”). }\end{array}$ & 2,39 & 0,74 & $-1,07$ & 0,78 \\
\hline 6. Es aceptado/a por otros niños. & 2,74 & 0,44 & $-1,13$ & $-0,73$ \\
\hline 7. Sigue las instrucciones de los adultos. & 2,60 & 0,55 & $-0,99$ & $-0,04$ \\
\hline 8. Intenta nuevas formas de resolver algo antes de pedir ayuda. & 2,13 & 0,81 & $-0,60$ & $-0,31$ \\
\hline 9. Hace amigos fácilmente. & 2,58 & 0,57 & $-0,98$ & $-0,02$ \\
\hline 11. Es invitado/a por otros niños para jugar. & 2,72 & 0,48 & $-1,39$ & 0,87 \\
\hline 13. Es capaz de separarse de sus padres sin una tristeza extrema. & 2,79 & 0,50 & $-2,40$ & 4,95 \\
\hline 14. Participa de las conversaciones en la clase. & 2,38 & 0,74 & $-0,98$ & 0,37 \\
\hline 15. Pide ayuda a los adultos cuando la necesita. & 2,50 & 0,60 & $-0,75$ & $-0,41$ \\
\hline 17. Defiende los derechos de otros niños (“iesto es de él!"). & 2,36 & 0,71 & $-0,82$ & 0,09 \\
\hline 18. Se adapta bien a diferentes ambientes. & 2,73 & 0,50 & $-1,69$ & 2,02 \\
\hline 19. Tiene cualidades o habilidades que son admiradas por otros niños. & 2,05 & 0,79 & $-0,21$ & $-1,01$ \\
\hline 20. Consuela a otros niños cuando están molestos. & 2,14 & 0,84 & $-0,65$ & $-0,38$ \\
\hline 21. Invita a otros niños a jugar. & 2,60 & 0,58 & $-1,15$ & 0,35 \\
\hline 22. Arregla su desorden cuando se lo piden. & 2,26 & 0,78 & $-0,82$ & 0,11 \\
\hline 23. Sigue las reglas. & 2,50 & 0,66 & $-1,20$ & 1,14 \\
\hline 24. Busca consuelo de los adultos cuando se hace daño. & 2,50 & 0,67 & $-1,00$ & $-0,20$ \\
\hline 25. Comparte juguetes u otras pertenencias. & 2,55 & 0,59 & $-0,89$ & $-0,18$ \\
\hline 26. Defiende sus propios derechos. & 2,65 & 0,58 & $-1,41$ & 1,00 \\
\hline $\begin{array}{l}\text { 27. Pide disculpas por un comportamiento accidental que puede } \\
\text { molestar a otros. }\end{array}$ & 2,28 & 0,80 & $-0,66$ & $-0,74$ \\
\hline 28. Negocia con sus compañeros cuando es apropiado. & 2,17 & 0,71 & $-0,35$ & $-0,62$ \\
\hline 29. Acepta decisiones de los adultos. & 2,67 & 0,57 & $-1,53$ & 1,35 \\
\hline 30. Intercambia juguetes u otros objetos. & 2,54 & 0,59 & $-0,85$ & $-0,26$ \\
\hline 31. Es seguro/a en situaciones sociales. & 2,47 & 0,61 & $-1,00$ & 1,35 \\
\hline 32. Reacciona de forma apropiada cuando le corrigen. & 2,61 & 0,58 & $-1,18$ & 0,41 \\
\hline 33. Es sensible a los problemas de los adultos (“¿estás triste?”). & 2,21 & 0,81 & $-0,41$ & $-1,35$ \\
\hline 34. Demuestra afecto a otros niños. & 2,53 & 0,63 & $-1,01$ & $-0,05$ \\
\hline
\end{tabular}

Nota. Los ítems 10, 12 y 16 fueron eliminados antes de este análisis. 
Tabla 2

Media, Desviación Estándar, Asimetría y Curtosis de la Distribución de los Ítems de la Escala de Problemas de Conducta

\begin{tabular}{|c|c|c|c|c|}
\hline Ítem & $M$ & $D E$ & Asimetría & Curtosis \\
\hline 1. Actúa impulsivamente y sin pensar. & 1,21 & 1,01 & 0,27 & $-1,06$ \\
\hline 2. Se enferma cuando está molesto/a o asustado/a. & 0,59 & 0,82 & 1,12 & 0,12 \\
\hline 3. Se burla de otros niños. & 0,62 & 0,85 & 1,02 & $-0,27$ \\
\hline 4. No responde al cariño de otros (por ej, un abrazo). & 1,15 & 0,96 & 0,20 & $-1,09$ \\
\hline 5. Se apega a los padres o cuidadores. & 1,01 & 0,99 & 0,66 & $-0,62$ \\
\hline 6. Hace alborotos que molestan a los demás. & 0,78 & 0,92 & 0,62 & $-1,14$ \\
\hline 7. Tiene arranques de enojo o berrinches. & 0,69 & 0,97 & 1,10 & $-0,12$ \\
\hline 8. Quiere toda la atención. & 1,12 & 1,10 & 0,50 & $-1,10$ \\
\hline 9. Es ansioso/a o tenso/a. & 0,98 & 1,06 & 0,70 & $-0,81$ \\
\hline 10. No comparte. & 1,05 & 0,85 & 0,21 & $-0,93$ \\
\hline 11. Es agresivo/a físicamente (pega, patea, empuja). & 0,76 & 0,93 & 1,03 & 0,07 \\
\hline 12. Evita jugar con otros niños. & 0,72 & 0,79 & 0,87 & 0,12 \\
\hline 13. Grita cuando está enojado/a. & 0,67 & 0,91 & 1,13 & 0,18 \\
\hline 14. Le quita cosas a otros niños. & 0,71 & 0,77 & 0,55 & $-1,09$ \\
\hline 16. Desobedece las reglas. & 1,03 & 0,99 & 0,40 & $-1,12$ \\
\hline 17. Tiene dificultades para hacer amigos. & 0,80 & 0,73 & 0,33 & $-1,08$ \\
\hline 18. Todo tiene que ser a su manera. & 1,10 & 0,98 & 0,26 & $-1,17$ \\
\hline 19. Es miedoso/a o asustadizo/a. & 0,73 & 0,82 & 0,70 & $-0,64$ \\
\hline 20. Es muy activo/a, no puede quedarse quieto/a. & 1,18 & 1,08 & 0,38 & $-1,16$ \\
\hline 21. Intenta vengarse de los demás. & 0,55 & 0,72 & 1,09 & 0,46 \\
\hline 23. Se queja de dolores, lastimaduras o enfermedades. & 0,58 & 0,67 & 0,75 & $-0,56$ \\
\hline 24. Se resiste a ir a la escuela. & 0,33 & 0,59 & 1,60 & 1,49 \\
\hline 25. Es inquieto/a. & 1,19 & 1,11 & 0,21 & $-1,42$ \\
\hline 26. Dice groserías a otros niños. & 0,39 & 0,69 & 1,49 & 0,75 \\
\hline 27. Es difícil de consolar cuando está molesto/a. & 0,73 & 0,72 & 0,46 & $-0,98$ \\
\hline 28. Se aleja de la compañía de los demás. & 0,55 & 0,67 & 0,82 & $-0,46$ \\
\hline 29. Intimida a otros niños. & 0,49 & 0,77 & 1,31 & 0,48 \\
\hline 30. Parece triste o deprimido/a. & 0,52 & 0,77 & 1,43 & 1,34 \\
\hline 31. Tiene un comportamiento impredecible. & 0,46 & 0,65 & 1,49 & 2,25 \\
\hline 32. Tiene celos de otros niños. & 0,35 & 0,57 & 1,40 & 0,98 \\
\hline 33. Actúa como si tuviera menos años. & 0,42 & 0,78 & 1,65 & 1,41 \\
\hline 34. Destruye las cosas que pertenecen a otros. & 0,34 & 0,61 & 1,62 & 1,47 \\
\hline 36. Es demasiado sensible a las críticas o castigos. & 1,39 & 1,00 & 0,40 & $-0,92$ \\
\hline 37. Es quejoso/a. & 0,66 & 0,93 & 1,20 & 0,29 \\
\hline 38. Otros niños se aprovechan de él/ella. & 0,39 & 0,71 & 2,05 & 4,14 \\
\hline 39. Perturba las actividades que se están desarrollando. & 0,85 & 0,97 & 0,54 & $-1,20$ \\
\hline 40. Dice mentiras. & 0,54 & 0,72 & 1,11 & 0,49 \\
\hline 41. Es provocado/a fácilmente, tiene poca paciencia. & 0,80 & 0,98 & 0,95 & $-0,30$ \\
\hline 42. Molesta a otros niños. & 0,79 & 0,92 & 0,72 & $-0,79$ \\
\hline
\end{tabular}

Nota. Los ítems 15, 22 y 35 fueron eliminados antes de este análisis. 
asimetría negativa, mientras que los ítems de la escala de Problemas de Conducta mostraron asimetría positiva. Se eliminaron los reactivos con valores altos de asimetría ( \pm 1,5) que pudieran afectar la distribución normal de las puntuaciones. Se eliminaron, por lo tanto, los siguientes ítems: 3, 13, 18 y 29 de la escala de Habilidades Sociales; y 24, 26, 31, 33, 34 y 38 de la escala de Problemas de Conducta.

Posteriormente se inspeccionaron las matrices de correlación y de correlación anti-imagen de cada una de las escalas. Coeficientes de correlación mayores a 0,30 en la matriz de correlación y valores bajos fuera de la diagonal en la matriz anti-imagen son indicadores de que la matriz se puede factorizar (Tabachnick \& Fidell, 2001). En la matriz de correlación de la escala de Habilidades Sociales el 37\% de los valores fueron mayores a 0,30 y en la escala de Problemas de Conducta, el 54\%. Las matrices de correlación anti-imagen presentaron valores pequeños fuera de la diagonal.

\section{Análisis de la Estructura Interna y Consistencia Interna}

Escala de Habilidades Sociales. Un total de 27 ítems fueron sometidos a un análisis de componentes principales, de la misma manera que en el estudio de la versión original (Merrell, 1996). El índice de adecuación muestral de Kaiser Meyer Olkin (KMO) fue 0,753 y el test de esfericidad de Bartlett fue significativo $\left(\chi^{2}\right.$ aprox. [351, $N$ $=192]=3081,787, p=0,000)$, lo cual indica la factibilidad del estudio factorial. Con la regla de Kaiser-Guttman se obtuvo una solución inicial de ocho factores con autovalores mayores a 1, que explicaban el 72,9\% de la varianza total de las habilidades sociales. En base a la interpretación del gráfico de sedimentación y del análisis paralelo de Horn, se determinó la extracción de tres factores, que fueron rotados mediante rotación Varimax. A partir de la inspección de la matriz de componentes y considerando las comunalidades, se eliminaron un ítem con carga factorial baja $(<0,40)$, tres ítems con cargas factoriales elevadas compartidas, un ítem que cargaba en un factor sin coherencia teórica y tres ítems que pre- sentaron valores bajos de comunalidad (< $0,30)$. Los tres factores explicaron el $56,6 \%$ de la varianza total de las habilidades sociales. El Factor 1 contiene ítems referidos a Interacción Social, el Factor 2, ítems referidos a Cooperación Social y el Factor 3 contiene ítems sobre Independencia Social (Tabla 3).

Todos los ítems presentaron comunalidades mayores al 40\%. Para estimar la consistencia interna de la escala total y de las subescalas se calculó el índice alfa de Cronbach. Cabe señalar que índices entre 0,70 y 0,80 se consideran buenas estimaciones de consistencia interna (Kaplan \& Saccuzzo, 2006), mientras que, como señala Loewenthal (2001), es aceptable un criterio menor (alrededor de 0,60) en escalas que cumplen los siguientes criterios: escaso número de ítems, buena evidencia de validez y razones prácticas o teóricas. En este sentido, como se puede apreciar en la Tabla 3 , la escala total y las subescalas mostraron altos índices de consistencia interna ( $\alpha=$ 0,$88 ; 0,84 ; 0,86$ y 0,74 , respectivamente).

Escala de Problemas de Conducta. Se utilizaron 33 ítems para el análisis de componentes principales. El índice de adecuación muestral KMO fue 0,855 y el test de esfericidad de Bartlett fue significativo $\left(\chi^{2}\right.$ aprox. $[528, N=192]=5020,106, p=$ $0,000)$, por lo cual era factible realizar el análisis factorial. Con la regla de KaiserGutman se obtuvo una solución inicial de siete factores con autovalores mayores a 1 , que explicaban el $69,8 \%$ de la varianza total de problemas de conducta. En base a la interpretación del gráfico de sedimentación y del análisis paralelo de Horn, se determinó la extracción de dos factores, rotados mediante rotación Varimax. A partir de la inspección de la matriz de componentes se eliminaron dos ítems que presentaban cargas factoriales menores a 0,40 , cuatro ítems con cargas factoriales compartidas elevadas, cuatro ítems que cargaban en un factor sin coherencia teórica y cinco ítems con valores de comunalidad menores a 0,30. Los dos factores explicaron el $62 \%$ de la varianza total de problemas de conducta. El Factor 1 contiene ítems referidos a Problemas Externalizantes y el Factor 2, 
Tabla 3

Estructura Factorial de la Escala de Habilidades Sociales

\begin{tabular}{|c|c|c|c|c|}
\hline \multirow{2}{*}{\multicolumn{2}{|c|}{ Ítem }} & \multicolumn{3}{|c|}{ Factor } \\
\hline & & 1 & 2 & 3 \\
\hline \multicolumn{2}{|c|}{ 5. Intenta comprender el comportamiento de otro niño (“¿por qué lloras?”). } & 0,84 & & \\
\hline \multicolumn{2}{|l|}{ 11. Es invitado/a por otros niños para jugar. } & 0,57 & & \\
\hline \multicolumn{2}{|c|}{ 17. Defiende los derechos de otros niños (“iesto es de él!”). } & 0,61 & & \\
\hline \multicolumn{2}{|l|}{ 20. Consuela a otros niños cuando están molestos. } & 0,76 & & \\
\hline \multicolumn{2}{|c|}{ 24. Busca consuelo de los adultos cuando se hace daño. } & 0,65 & & \\
\hline \multicolumn{2}{|c|}{ 28. Negocia con sus compañeros cuando es apropiado. } & 0,57 & & \\
\hline \multicolumn{2}{|c|}{ 33. Es sensible a los problemas de los adultos (“¿estás triste?”). } & 0,75 & & \\
\hline \multicolumn{2}{|l|}{ 34. Demuestra afecto a otros niños. } & 0,57 & & \\
\hline \multicolumn{2}{|l|}{ 2. Es cooperativo/a. } & \multicolumn{3}{|c|}{0,52} \\
\hline \multicolumn{2}{|l|}{ 7. Sigue las instrucciones de los adultos. } & \multicolumn{3}{|c|}{0,82} \\
\hline \multicolumn{2}{|l|}{ 22. Arregla su desorden cuando se lo piden. } & \multicolumn{3}{|c|}{0,73} \\
\hline \multicolumn{2}{|l|}{ 23. Sigue las reglas. } & \multicolumn{3}{|c|}{0,83} \\
\hline \multicolumn{2}{|l|}{ 25. Comparte juguetes u otras pertenencias. } & \multicolumn{3}{|c|}{0,67} \\
\hline \multicolumn{2}{|l|}{ 30. Intercambia juguetes u otros objetos. } & \multicolumn{3}{|c|}{0,61} \\
\hline \multicolumn{2}{|c|}{ 32. Reacciona de forma apropiada cuando le corrigen. } & \multicolumn{3}{|c|}{0,69} \\
\hline \multicolumn{2}{|l|}{ 4. Juega con diferentes niños. } & & & 0,68 \\
\hline \multicolumn{2}{|l|}{ 9. Hace amigos fácilmente. } & & & 0,79 \\
\hline \multicolumn{2}{|l|}{ 21. Invita a otros niños a jugar. } & & & 0,51 \\
\hline \multicolumn{2}{|l|}{ 31. Es seguro/a en situaciones sociales. } & & & 0,75 \\
\hline \multirow{4}{*}{$\begin{array}{l}\text { KMO }=0,776 \\
\text { Test de Bartlett: } \chi^{2}(171, N=192)=1906,088 \\
p=0,000\end{array}$} & Varianza explicada & $22,81 \%$ & $20,06 \%$ & $13,75 \%$ \\
\hline & Varianza total explicada & & $56,62 \%$ & \\
\hline & alfa de Cronbach & 0,84 & 0,86 & 0,74 \\
\hline & alfa de Cronbach total & & 0,88 & \\
\hline
\end{tabular}

ítems referidos a Problemas Internalizantes (Tabla 4).

La comunalidad de los ítems fue mayor al $40 \%$. Con respecto a la consistencia interna y en función de los criterios señalados anteriormente, el alfa de Cronbach de la escala total $(0,94)$ y del primer factor $(0,96)$ fueron muy altos, en tanto fue relativamente bajo para el segundo factor $(0,67)$.

La versión final de la escala de Habilidades Sociales resultó compuesta por 19 ítems, 8 ítems correspondientes a Interacción Social, 7 ítems de Cooperación Social y 4 ítems de Independencia Social. La escala de Problemas de Conducta, por su parte, quedó formada por 18 ítems, 13 ítems de Problemas Externalizantes y 5 ítems de Problemas Internalizantes. De la misma manera que en la versión original del instrumento, mayor puntuación en la escala o subescalas de Habilidades Sociales señala un mejor desarrollo social; por el contrario, mayor puntuación en Problemas de Conducta indica comportamientos sociales negativos. 
Tabla 4

Estructura Factorial de la Escala de Problemas de Conducta

\begin{tabular}{|c|c|c|c|}
\hline \multirow{2}{*}{\multicolumn{2}{|c|}{ Ítem }} & \multicolumn{2}{|c|}{ Factor } \\
\hline & & 1 & 2 \\
\hline \multicolumn{2}{|l|}{ 1. Actúa impulsivamente y sin pensar. } & 0,83 & \\
\hline \multicolumn{2}{|l|}{ 3. Se burla de otros niños. } & 0,78 & \\
\hline \multicolumn{2}{|l|}{ 6. Hace alborotos que molestan a los demás. } & 0,84 & \\
\hline \multicolumn{2}{|l|}{ 7. Tiene arranques de enojo o berrinches. } & 0,83 & \\
\hline \multicolumn{2}{|l|}{ 11. Es agresivo/a físicamente (pega, patea, empuja). } & 0,81 & \\
\hline \multicolumn{2}{|l|}{ 14. Le quita cosas a otros niños. } & 0,78 & \\
\hline \multicolumn{2}{|l|}{ 16. Desobedece las reglas. } & 0,83 & \\
\hline \multicolumn{2}{|l|}{ 20. Es muy activo/a, no puede quedarse quieto/a. } & 0,81 & \\
\hline \multicolumn{2}{|l|}{ 21. Intenta vengarse de los demás. } & 0,68 & \\
\hline \multicolumn{2}{|l|}{ 25. Es inquieto/a. } & 0,83 & \\
\hline \multicolumn{2}{|c|}{ 39. Perturba las actividades que se están desarrollando. } & 0,84 & \\
\hline \multicolumn{2}{|c|}{ 41. Es provocado/a fácilmente, tiene poca paciencia. } & 0,77 & \\
\hline \multicolumn{2}{|l|}{ 42. Molesta a otros niños. } & 0,87 & \\
\hline \multicolumn{2}{|l|}{ 2. Se enferma cuando está molesto/a o asustado/a. } & & 0,60 \\
\hline \multicolumn{2}{|l|}{ 5. Se apega a los padres o cuidadores. } & & 0,55 \\
\hline \multicolumn{2}{|l|}{ 17. Tiene dificultades para hacer amigos. } & & 0,69 \\
\hline \multicolumn{2}{|l|}{ 28. Se aleja de la compañía de los demás. } & & 0,67 \\
\hline \multicolumn{2}{|l|}{ 30. Parece triste o deprimido/a. } & & 0,66 \\
\hline \multirow{4}{*}{$\begin{array}{l}\text { KMO }=0,915 \\
\text { Test de Bartlett: } \chi^{2}(153, N=192)=2630,498 \\
p=0,000\end{array}$} & Varianza explicada & $48,35 \%$ & $13,64 \%$ \\
\hline & Varianza total explicada & \multicolumn{2}{|c|}{$61,99 \%$} \\
\hline & alfa de Cronbach & 0,96 & 0,67 \\
\hline & alfa de Cronbach total & \multicolumn{2}{|c|}{0,94} \\
\hline
\end{tabular}

\section{Análisis Factorial Confirmatorio}

Se llevaron a cabo análisis factoriales confirmatorios (AFC) para valorar la estructura dimensional de la escala de Habilidades Sociales (trifactorial) y de la escala de Problemas de Conducta (bifactorial). Los casos incluidos en estos análisis no presentaron valores omitidos. Se utilizó el método de estimación de mínimos cuadrados no ponderados (ULS, por sus siglas en inglés), de la misma manera que en los estudios originales de la escala (Merrell, 1996). Una de las características del método de ULS es que no requiere distribuciones normales (Schumacker \& Lomax, 2004), por lo que resulta óptimo para reactivos comportamentales que generalmente presentan asimetría. Siguiendo las recomendaciones de Jöreskog (2002/2005) para el análisis de variables ordinales, tales como los ítems de la escala bajo estudio, los análisis se realizaron en base a las correlaciones policóricas y la matriz de covarianzas asintóticas, lo que permite corregir los errores estándar y el valor de $\chi^{2}$ cuando no hay normalidad. Además, se permitieron correlaciones al interior de cada una de las subescalas. 
Para evaluar el ajuste de los modelos se recurrió a indicadores de ajuste absoluto: $\chi^{2}$, índice de bondad del ajuste (GFI) y su variante ajustada (AGFI) y residuo cuadrático medio estandarizado (SRMR); a indicadores de ajuste comparativo: índice de ajuste comparativo (CFI) e índice de ajuste no normado (NNFI); y a indicadores de bondad de ajuste que penalizan la ausencia de parsimonia: error cuadrático medio de aproximación (RMSEA), índice de ajuste normado de parsimonia (PNFI) e índice de bondad de ajuste de parsimonia (PGFI) (Brown, 2006; Hu \& Bentler, 1999; Schumacker \& Lomax, 2004). Si bien el estadístico $\chi^{2}$ indica el ajuste absoluto del modelo, es sensible al tamaño de la muestra y a violaciones del supuesto de normalidad multivariada, por lo que se recomienda considerar además otros índices de ajuste.

Los resultados del AFC indican que el modelo de Habilidades Sociales trifactorial, según el estadístico $\chi^{2}([123, N=184]=$ $890,65, p=0,000)$, no ofrece un buen ajuste. Siguiendo las recomendaciones de considerar múltiples indicadores, se observó que el modelo presenta un ajuste adecuado según los criterios señalados de ajuste absoluto $(\mathrm{GFI}=0,99 ; \mathrm{AGFI}=0,98 ; \mathrm{SRMR}=0,07)$, de comparación con el modelo independiente $(\mathrm{CFI}=1,00$; NNFI $=0,99)$ y medidas que consideran la parsimonia (RMSEA $=0,03$; PNFI $=0,71 ;$ PGFI $=0,64)($ Tabla 5$)$. Los pesos de regresión estandarizados en el factor Interacción oscilaron entre 0,27 y 0,85, en Cooperación, entre 0,66 y 0,91 y en Independencia, entre 0,64 y 0,87 .

Por otra parte, para el modelo bifactorial de Problemas de Conducta, el AFC indica un muy buen ajuste según las medidas de ajuste absoluto $\left(\chi^{2}[131, N=184]=112,66\right.$, $p=0,875$; GFI = 0,99; AGFI 0,99; SRMR $=0,06)$, las medidas de comparación con el modelo independiente $(\mathrm{CFI}=1,00 ; \mathrm{NNFI}$ $=1,00)$ y las medidas que comprenden la parsimonia $(\mathrm{RMSEA}=0,00$; $\mathrm{PNFI}=0,85$; PGFI $=0,76$ ) (Tabla 5). Los pesos de regresión estandarizados en el factor Problemas Externalizantes oscilaron entre 0,78 y 0,94 y en el factor Problemas Internalizantes, entre 0,48 y 0,91 .

Tabla 5

Índices de Ajuste del Modelo de Habilidades Sociales y del Modelo de Problemas de Conducta

\begin{tabular}{lcc}
\hline & \multicolumn{2}{c}{ Modelo } \\
\cline { 2 - 3 } Índice de Ajuste & $\begin{array}{c}\text { Habilidades Sociales } \\
3 \text { factores }\end{array}$ & $\begin{array}{c}\text { Problemas de Conducta } \\
2 \text { factores }\end{array}$ \\
\hline$\chi^{2}$ & 890,65 & 112,66 \\
GFI & 0,99 & 0,99 \\
AGFI & 0,98 & 0,99 \\
SRMR & 0,07 & 0,06 \\
CFI & 1,00 & 1,00 \\
NNFI & 0,99 & 1,00 \\
RMSEA & 0,03 & 0,00 \\
PNFI & 0,71 & 0,85 \\
PGFI & 0,64 & 0,76 \\
\hline
\end{tabular}

Nota GFI = Índice de bondad del ajuste; AGFI = Índice de bondad del ajuste ajustado; SRMR = Residuo cuadrático medio estandarizado; CFI = Índice de ajuste comparativo; NNFI = Índice de ajuste no normado; RMSEA = Error cuadrático medio de aproximación; PNFI = Índice de ajuste normado de parsimonia; PGFI = Índice de bondad de ajuste de parsimonia; $N=184$. 
Discusión

A partir del estudio instrumental realizado a la Escala de Comportamiento Preescolar y Jardín Infantil se obtuvo una versión reducida de la escala, con adecuadas propiedades psicométricas, por lo cual se considera un buen instrumento para la evaluación del comportamiento social en niños de 3 a 7 años.

La escala general comprende dos grandes subescalas: una correspondiente a Habilidades Sociales y otra, a Problemas de Conducta. La escala de Habilidades Sociales mostró una estructura factorial semejante a la obtenida en estudios de la versión original (Merrell, 1996), con tres factores: Interacción Social, Cooperación Social e Independencia Social. Aunque en el presente estudio se obtuvo una versión con menor cantidad de reactivos, los tres factores explicaron el $56,62 \%$ de la varianza de habilidades sociales. Algunos ítems cargaron en factores distintos a los propuestos por el autor de la versión original; por ejemplo, el ítem "Negocia con sus compañeros cuando es apropiado" correspondía a Cooperación Social en el modelo original y en este estudio carga en el factor Interacción Social. Considerando que existe adecuada coherencia teórica, se optó por mantener el ítem en el modelo. En el análisis confirmatorio se evaluaron múltiples indicadores, obteniéndose un ajuste adecuado para el modelo trifactorial de Habilidades Sociales, lo cual aboga a favor de la estabilidad del modelo. Los índices de consistencia interna fueron altos, con alfa de Cronbach entre 0,74 y 0,88.

Con respecto a la escala de Problemas de Conducta, la solución factorial fue semejante a la obtenida con la versión original (Merrell, 1996), la cual presentaba una estructura bifactorial: Problemas Externalizantes e Internalizantes. En este estudio, en el que la cantidad de ítems resultante fue menor, los dos factores explicaron el $61,99 \%$ de la varianza total de problemas de conducta. Además, el análisis confirmatorio arrojó un muy buen ajuste del modelo de dos factores de Problemas de Conducta, lo cual indica la estabilidad de su estructura. En cuanto a la confiabilidad, se obtuvieron muy altos índices de consistencia interna de la esca- la total y Problemas Externalizantes (alfa de Cronbach 0,94 y 0,96, respectivamente). Sin embargo, la subescala de Problemas Internalizantes presentó un índice relativamente bajo de consistencia interna (alfa de Cronbach 0,67), probablemente debido al bajo número de ítems resultante (cinco).

Como se señaló anteriormente, este estudio se enmarca en un proyecto mayor sobre desarrollo social infantil. En tal investigación los docentes informan, entre otros aspectos, sobre el comportamiento social, mientras que los padres aportan información sobre emocionalidad y regulación emocional. En estudios preliminares tanto padres como docentes reportaron el comportamiento social; sin embargo, la cantidad de instrumentos que debían completar los padres generaba fatiga y oposición, por lo cual, considerando que los docentes son señalados en la literatura como los mejores informantes, los estudios se continuaron solo con su reporte. El haber recurrido solo a un informante constituye una de las limitaciones de este estudio, lo que se suma a una característica inherente de las escalas de evaluación, las cuales miden la percepción del evaluador sobre la conducta del niño más que la conducta en sí. Se destaca la necesidad de contar con el reporte de los padres, lo que aportará evidencia del comportamiento del niño en otro contexto y brindará una herramienta de evaluación para niños no escolarizados. Se espera en estudios futuros recurrir a diversas técnicas e informantes, lo que permitirá lograr una mejor comprensión del comportamiento infantil.

En ese sentido, el estudio conjunto del comportamiento social con varios instrumentos de evaluación aportará evidencia de validez concurrente. Asimismo, teniendo en cuenta las relaciones señaladas entre el desarrollo social en edades tempranas y el comportamiento social, desempeño académico y problemas psicopatológicos futuros, entre otros, estudios que relacionen tales aspectos significarán un valioso aporte de validez predictiva.

Como señala Merrell (1996), es necesario avanzar en la conceptualización, evaluación e intervención de los problemas socioemocionales. Resulta de interés contar 
con estudios normativos sobre el desarrollo social, para lo cual se necesitará seleccionar muestras mayores y obtenidas de modo probabilístico. De esta manera se podrán establecer puntos de corte para identificar, en situaciones de screening, potenciales problemas de comportamiento y desarrollo socioemocional, tomando como referencia una muestra normativa. Además, es necesario comprender de manera integrada el desarrollo social, lo que implica estudiar las interrelaciones entre aspectos sociales, cognitivos y emocionales. Ello permitirá implementar mejores estrategias de prevención e intervención.

Si bien en este trabajo resultaron soluciones semejantes a las obtenidas en el estudio de la versión original y con adecuadas propiedades psicométricas, las diferencias señaladas dan cuenta de la importancia de adaptar los instrumentos elaborados en países de lengua y cultura diferentes a la población de interés (Hambleton, 2001; Poortinga, 2000).

\section{Referencias}

Argentina, Ministerio de Salud de la Nación y Unidades Académicas de Psicología de Universidades Nacionales (2007). Problemáticas de salud mental en la infancia. Proyecto de investigación. Informe final. Buenos Aires: Autores. Extraído el 23 Julio, 2008, del sitio web del Ministerio de Salud de la Nación: http://www.msal.gov.ar/htm/site/pdf/ informe_final.pdf

Bank, L., Duncan, T., Patterson, G. R. \& Reid, J. (1993). Parent and teacher ratings in the assessment and prediction of antisocial and delinquent behaviors. Journal of Personality, 61, 693-709.

Brown, T. A. (2006). Confirmatory factor analysis for applied research. New York: The Guilford Press.

Brussino, S.A. (2002). Análisiscausaldel comportamiento agresivo infantil: pautas de crianza, estilo atribucional, capacidad intelectual y habilidad social. Tesis no publicada para optar al grado de Doctor en Psicología, Universidad Nacional de Córdoba, Argentina.

Caballo, V. (2005). Manual de evaluación y entrenamiento de las habilidades sociales $\left(3^{\mathrm{a}}\right.$ ed.). Madrid: Siglo XXI.

Caldarella, P. \& Merrell, K. W. (1997). Common dimensions of social skills of children and adolescents: A taxonomy of positive behaviors. School Psychology Review, 26, 264-278.

Canivez, G. L. \& Bordenkircher, S. E. (2002). Convergent and divergent validity of the adjustment scales for children and adolescent and preschool and kindergarten behavior scales. Journal of Psychoeducational Assessment, 20, 30-45.
Canivez, G. L. \& Rains, J. D. (2002). Construct validity of the Adjustment Scales for Children and Adolescents and the Preschool and Kindergarten Behavior Scales: Convergent and divergent evidence. Psychology in the Schools, 39, 621633.

Carney, A. G. \& Merrell, K. W. (2002). Reliability and comparability of a Spanish-language form of the Preschool and Kindergarten Behavior Scales. Psychology in the Schools, 39, 367-373.

Carney, A. G. \& Merrell, K. W. (2005). Teacher ratings of young children with and without ADHD: Construct validity of two child behavior rating scales. Assessment for Effective Intervention, 30(3), 65-75.

Cummings, K. D., Kaminski, R. A. \& Merrell, K. W. (2008). Advances in the assessment of social competence: Findings from a preliminary investigation of a general outcome measure for social behavior. Psychology in the Schools, 45, 930-946.

Gresham, F. M., Sugai, G. \& Horner, R. H. (2001). Interpreting outcomes of social skills training for students with high-incidence disabilities. Exceptional Children, 67, 331-344.

Hair, J. F., Anderson, R. E., Tatham, R. L. \& Black, W. C. (1999). Análisis multivariante (5 ${ }^{\mathrm{a}}$ ed.). Madrid: Prentice Hall.

Hambleton, R. K. (2001). The next generation of the ITC test translation and adaptation guidelines. European Journal of Psychological Assessment, 17, 164-172.

Holland, M. L. \& Merrell, K. W. (1998). Socio-emotional characteristics of preschool-aged children referred for child find screening and assessment: A comparative study. Research in Developmental Disabilities, 19, 167-179.

Hu, L. \& Bentler, P. M. (1999). Cutoff criteria for fit indexes in covariance structure analysis: Conventional criteria versus new alternatives. Structural Equation Modeling, 6, 1-55.

Ison, M. S. \& Facchinelli, C. C. (1993). Guía de observación comportamental para niños. Interdisciplinaria, 12, 11-21.

Ison, M. S. \& Soria, R. (1997). Baremo de la guía de observación comportamental para niños. Interdisciplinaria, 14, 25-46.

Jentzsch, C. E. \& Merrell, K. W. (1996). An investigation of the construct validity of the Preschool and Kindergarten Behavior Scales. Assessment for Effective Intervention, 21(2), 1-15.

Jöreskog, K. G. (2002/2005). Structural equation modeling with ordinal variables using LISREL. Lincolnwood, IL: Scientific Software International. Extraído el 10 Mayo, 2009, de http://www. ssicentral.com/lisrel/techdocs/ordinal.pdf

Kaplan, R. M. \& Saccuzzo, D. P. (2006). Pruebas psicológicas: principios, aplicaciones y temas $\left(6^{\mathrm{a}}\right.$ ed.). Madrid: International Thomson.

Lacunza, A. B., Castro Solano, A. \& Contini, E. N. (en prensa). Las habilidades sociales preescolares: construcción y validación de una escala para niños de contextos de pobreza. Psicología, Pontificia Universidad Católica del Perú.

Loewenthal, K. M. (2001). An introduction to psychological tests and scales ( $2^{\text {nd }}$ ed.). London: 
Psychology Press.

Matson, J. L., Rotatori, A. F. \& Helsel, W. J. (1983). Development of a rating scale to measure social skills in children: The Matson Evaluation of Social Skills with Youngsters (MESSY). Behaviour Research and Therapy, 21, 335-340.

Matson, J. L. \& Wilkins, J. (2009). Psychometric testing methods for children's social skills. Research in Developmental Disabilities, 30, 249-274.

Merrell, K. W. (1996). Social-emotional assessment in early childhood: The Preschool and Kindergarten Behavior Scales. Journal of Early Intervention, 20, 132-145.

Merrell, K. W. (2000). Informant reports: Rating scale measures. En E. S. Shapiro \& T. K. Kratochwill (Eds.), Conducting school-based assessments of child and adolescent behavior (pp. 203-234). New York: The Guilford Press.

Merrell, K. W. (2003). Preschool and Kindergarten Behavior Scales, second edition: Spanish language version. Austin, TX: PRO-ED.

Merrell, K. W. \& Harlacher, J. E. (2008). Behavior rating scales. En R. P. Archer \& S. R. Smith (Eds.), Personality assessment (pp. 247-280). New York: Routledge/Taylor \& Francis.

Merrell, K. W. \& Wolfe, T. M. (1998). The relationship of teacher-rated social skills deficits and ADHD characteristics among kindergarten-age children. Psychology in the Schools, 35, 101-109.

Parker, J. G. \& Asher, S. R. (1987). Peer relations and later personal adjustment: Are low-accepted children at risk? Psychological Bulletin, 102, 357389.

Patterson, G. R., DeGarmo, D. S. \& Knutson, N. (2000). Hyperactive and antisocial behaviors: Comorbid or two points in the same process? Development and Psychopathology, 12, 91-106.

Poortinga, Y. H. (2000). Cross-cultural test adaptation.
En A. E. Kazdin (Ed.), Encyclopedia of psychology (pp. 369-371). Washington, DC: American Psychological Association.

Rose-Krasnor, L. (1997). The nature of social competence: A theoretical review. Social Development, 6, 111-135.

Samaniego, V. C. (1998). El Child Behavior Check List: su estandarización y aplicación en un estudio epidemiológico. Problemas comportamentales y sucesos de vida en niños de 6 a 11 años de edad (Informe Final UBACYT). Buenos Aires: Universidad de Buenos Aires, Facultad de Psicología.

Schulz, A. (2008). Validación de un sistema de evaluación de las habilidades sociales en niños argentinos por medio de informantes clave. Resultados preliminares (Informe final correspondiente al Proyecto de Investigación 26/07 de la Facultad de Humanidades, Educación y Ciencias Sociales). Libertador San Martín, Argentina: Universidad Adventista del Plata.

Schumacker, R. E. \& Lomax, R. G. (2004). A beginner's guide to structural equation modeling ( $2^{\text {nd }}$ ed.). Mahwah, NJ: Lawrence Erlbaum Associates.

Stanger, C. \& Lewis, M. (1993). Agreement among parents, teachers, and children on internalizing and externalizing behavior problems. Journal of Clinical Child Psychology, 22, 107-115.

Tabachnick, B. \& Fidell, L. (2001). Using multivariate statistics $\left(4^{\text {th }}\right.$ ed.). New York: Harper \& Row.

Trianes, M. V., Muñoz, A. M. \& Jiménez, M. (1997). Competencia social: su educación y tratamiento. Madrid: Pirámide.

Winsler, A. \& Wallace, G. L. (2002). Behavior problems and social skills in preschool children: Parentteacher agreement and relations with classroom observations. Early Education and Development, 13, 41-58.

Fecha de recepción: Marzo de 2009.

Fecha de aceptación: Septiembre de 2009. 